\title{
Conductivity Variation during Irreversible Electroporation
}

\author{
Chao $\mathrm{Xu}^{1}$, Lei Yang ${ }^{1, *}$ \\ ${ }^{1}$ China Academy of Information and Communications Technology, Beijing, 100191, China
}

\begin{abstract}
Irreversible electroporation (IRE) is an emerging tumour ablation technique, which utilizes non thermoelectric pulse to ablate tumours. Compared with the common tumour treatment methods, e.g., surgery, chemotherapy and radiotherapy, IRE has advantages of short action time, no massive bleeding of tissue during ablation, avoiding heat sink effect, etc. However, the process of IRE will be affected by the distribution of E-field of the tissue. The major factors which affect E-field distribution are parameters of the electrical pulse and characteristics of the tissue, e.g., the conductivity of the tissue. We utilized the finite element analysis software COMSOL Multiphysics 5.4 to calculate the variation in liver tissue conductivity during IRE. In this study, there is a Low-Voltage High-Frequency (LVHF) pulse involves 3500 unipolar and bipolar pulses, and a High-Voltage Low-Frequency (HVLF) pulse involves eight unipolar and bipolar pulses. The results show that the conductivity change of the HVLF pulse is higher than that of the LVHF pulse due to the higher strength of E-field. The most important conclusion is that the LVHF pulse can only change the tissue conductivity near the electrode tip. The HVLF pulse will significantly change the conductivity in the tissue between the electrodes.
\end{abstract}

\section{Introduction}

Irreversible electroporation is achieved by applying a series of short-duration and high-amplitude electric pulses to the target tissue, and forming a high-strength Efield in it to change the permeability of the cell membrane. It is a technique that does not rely on thermal energy for tissue ablation. The cell membrane structure is irreversibly damaged and the physiological balance of the cell is broken when the E-field intensity is high enough, then the cell is death and the purpose of ablating the tissue is achieved [1]. According to the existing research results, IRE is widely used in animal tissue ablation [2-3], angioplasty [4] and food sterilization [5]. In addition, IRE can also be used for tumour cell ablation, providing a new method for tumour treatment [6-7]. Since the mechanism of cell death does not depend on extreme temperature, irreversible electroporation can be safely performed near major blood vessels and nerves. Therefore, it is a feasible choice for unresectable tumours and functional limited resection. IRE has been used to treat liver, kidney, pancreas, prostate and other tumours [8-10].

Two IRE pulses are mentioned in this study, LowVoltage High-Frequency (LVHF) and High-Voltage Low-Frequency (HVLF). Patients who have been applied HVLF could feel pain, which may be caused by muscle contraction during each pulse [11]. However, the nerve stimulation threshold that causes muscle contraction will increase when the pulse frequency increases [12]. Therefore, the LVHF pulse produces a tiny heat in the tissue. Furthermore, it also greatly reduces the ache of patients during clinical application [13-14].

A large number of factors may affect the electroporation process, e.g., E-field intensity, pulse frequency, period, duration, pulse shape, number of electric pulses, and E-field distribution [15]. Among them, the E-field and temperature distribution are affected by electrical conductivity [16]. Different studies have shown that the conductivity increases during IRE [17-18]. And the measurement of tissue conductivity can optimize the efficiency of IRE programmes and predictions of the IRE process [19-20]. Needle electrodes have the advantages of flexible placement and the ability to treat superficial and deep tissues during IRE [21]. Therefore, we studied the influence of the needle electrode on the change of tissue conductivity during IRE electroporation. The focus of this study is to evaluate the variation in conductivity of the needle electrode during the LVHF and HVLF pulses.

\section{Methods and materials}

\subsection{Design of finite element model}

The finite element analysis software COMSOL Multiphysics 5.4 is utilized to calculate the finite element model in this study. The liver tissue was modelled as a cubic geometry. The diameters of the liver are $34 * 34 * 18 \mathrm{~mm} .6$ needle electrodes were used in the simulation. And these electrodes are distributed in two parallel rows. Each row of electrodes contains 3 electrodes. The structure diagram of the model is shown

\footnotetext{
* Corresponding author: yanglei@caict.ac.cn
} 
in Figure 1a. The diameter of each needle is $0.43 \mathrm{~mm}$, and the distance between three consecutive electrodes is $2.6 \mathrm{~mm}$, and the distance between two rows of electrodes is $8.66 \mathrm{~mm}$. In the COMSOL Multiphysics software, a symmetric model can be used to reduce the simulation time. Therefore, we created the symmetrical model (Figure 1b).
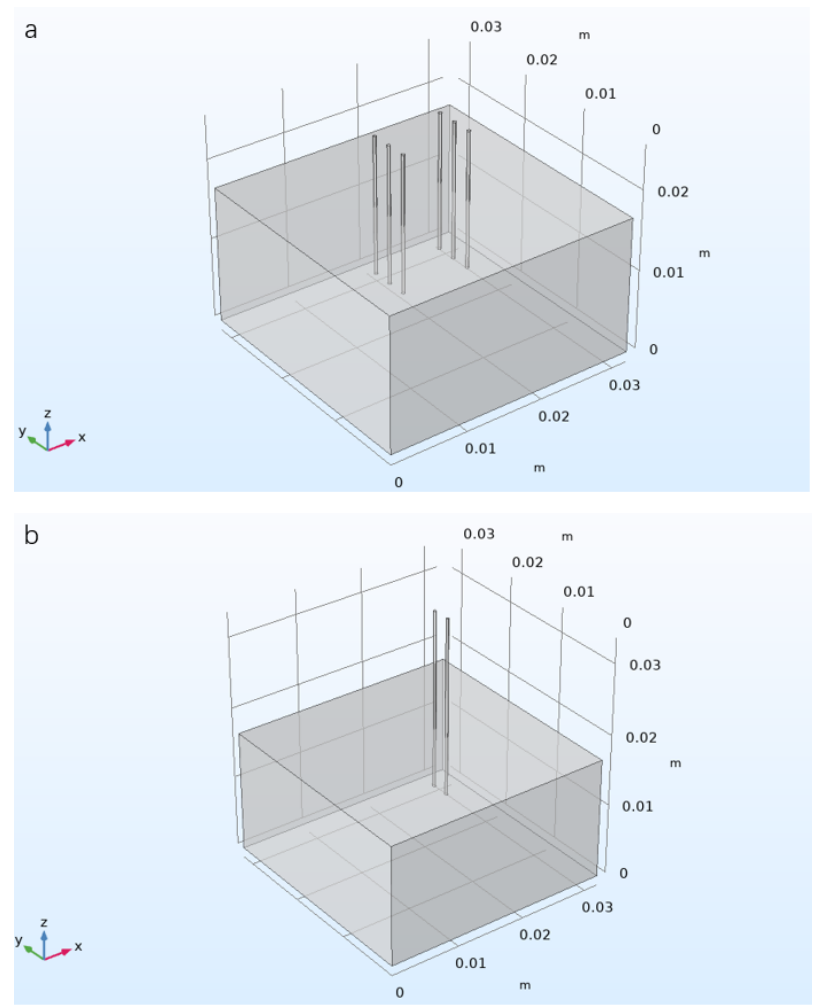

Fig. 1. The geometry of numerical modeling

\subsection{Model parameter setting}

Two electrical pulse parameters were used in this study, LVHF and HVLF pulses. Some researches show that IRE can be achieved by using LVHF pulses [22-23]. The LVHF pulse involves 3500 unipolar and bipolar pulses whose frequency, pulse width and E-field strength is 5.2 $\mathrm{kHz}, 0.1 \mathrm{~ms}$ and $300 \mathrm{~V} / \mathrm{cm}$, respectively. Unlike unipolar pulses, the maximum applied voltage in bipolar pulses is less than that of unipolar pulses. In addition, the HVLF pulse involves eight unipolar and bipolar pulses whose frequency, pulse width and E-field strength is $1 \mathrm{~Hz}, 2 \mathrm{~ms}$ and $3000 \mathrm{~V} / \mathrm{cm}$, respectively.

Table 1 shows that the electrical and thermal properties of liver electrodes and stainless-steel electrodes are listed in [24-25].

\subsection{Calculating method}

The E-field and potential distribution inside the tissue can obtained by Laplace equation:

$$
\vec{\nabla} \cdot(\sigma \cdot \vec{\nabla} \varphi)=0
$$

Where, $\sigma$ is conductivity of the tissue, and $\varphi$ is electric potential. Using Penne's Bioheat equation can estimate heat transfer in tissue:

$$
\nabla \cdot(k \nabla T)+\sigma|\nabla \varphi|^{2}+q^{\prime \prime \prime}-W_{b} c_{b} T=\rho c_{p} \frac{\partial T}{\partial t}
$$

Where, $\varphi$ is electrical potential, $T$ is temperature, $q^{\prime \prime \prime}$ is the heat produced by metabolism, $W_{b} c_{b} T$ is the heat produced by perfusion, $\rho$ is density, and $c_{p}$ is a specific heat capacity of tissue. The calculation equation for the change of conductivity inside the tissue during IRE is as follows:

$$
\begin{aligned}
\sigma= & \sigma_{0} *\left(1+f l c 2 h s\left(E-E_{\text {delta }}, E_{\text {range }}\right)\right. \\
& \left.+\alpha *\left(T-T_{0}\right)\right)
\end{aligned}
$$

Where, $\sigma_{0}$ is initial conductivity of the tissue, $E$ is the E-field, $E_{\text {delta }}$ is an E-field threshold, $E_{\text {range }}$ is E-field range, $\alpha$ is temperature coefficient, $T$ is temperature of the tissue, and $T_{0}$ is initial temperature of the tissue.

At the same time, for the purpose of ensuring good convergence of the numerical solution, we utilized flc $2 h s$ function which is a smoothed Heaviside function in COMSOL. The characteristic of the function is that the value changes from 0 to 1 while $E-E_{\text {delta }}=0$ is over the range $E_{\text {range }}$ [24]. The parameters which were utilized in Equation (3) are listed in Table 2 [24-25].

Table 1. The electric and thermal properties of the liver and stainless-steel electrodes.

\begin{tabular}{|c|c|c|}
\hline & electrode & liver \\
\hline $\begin{array}{c}\text { Electrical conductivity } \\
\text { (S/m) }\end{array}$ & $1.398 * 10^{6}$ & $\begin{array}{c}0.067 \\
\text { (initial) }\end{array}$ \\
\hline $\begin{array}{c}\text { Thermal conductivity } \\
\text { (W/m.K) }\end{array}$ & 16.3 & 0.512 \\
\hline Density (kg/m3) & 7800 & 1050 \\
\hline Heat capacity (J/kg.K) & 490 & 360 \\
\hline
\end{tabular}

Table 2. Parameters of simulation.

\begin{tabular}{|c|c|}
\hline Variables & Values \\
\hline$\sigma_{0}$ & $0.067(\mathrm{~S} / \mathrm{m})$ \\
\hline$E_{\text {delta }}$ & $580(\mathrm{~V} / \mathrm{cm})$ \\
\hline$E_{\text {range }}$ & $(-120,120)(\mathrm{V} / \mathrm{cm})$ \\
\hline$\alpha$ & $0.015\left({ }^{\circ} \mathrm{C}^{-1}\right)$ \\
\hline$T_{0}$ & $37\left({ }^{\circ} \mathrm{C}\right)$ \\
\hline
\end{tabular}

$\varphi=V(t)$ is the condition of electric boundary which belongs to active row. Where, $V(t)$ is a voltage pulse that changes with time. The remaining boundaries are considered electrically insulated. 


\section{Result and Discussion}

\subsection{Simulation results for conductivity variation in HVLF}

During the pulse transmission time, the conductivity variations of HVLF pulse which consists of 8 unipolar pulses is shown in Figure 2 and Figure 3. And the conductivity variations of HVLF pulse which consists of 8 bipolar pulses are shown in Figure 4 and Figure 5. Figure 2 shows the conductivity variations at the tip of the middle electrode in a row of electrodes, where HVLF pulses were applied to the entire electrode. And Figure 3 shows the variations in conductivity between the two rows of electrodes. Figures 4 and 5 show these results for bipolar HVLF pulses.

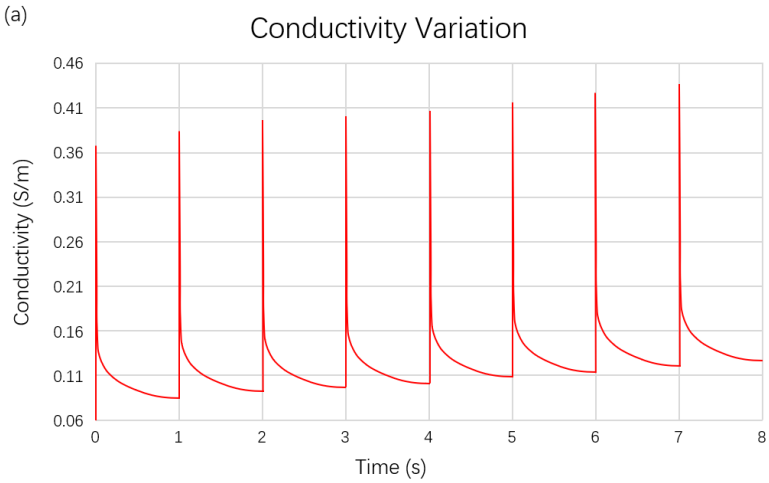

Fig. 2. Conductivity variation at the tip of the needle electrodes under the action of 8 unipolar pulses whose frequency, pulse width, and E-field strength is $1 \mathrm{~Hz}, 2 \mathrm{~ms}$, and $3000 \mathrm{~V} / \mathrm{cm}$, respectively.

(b)

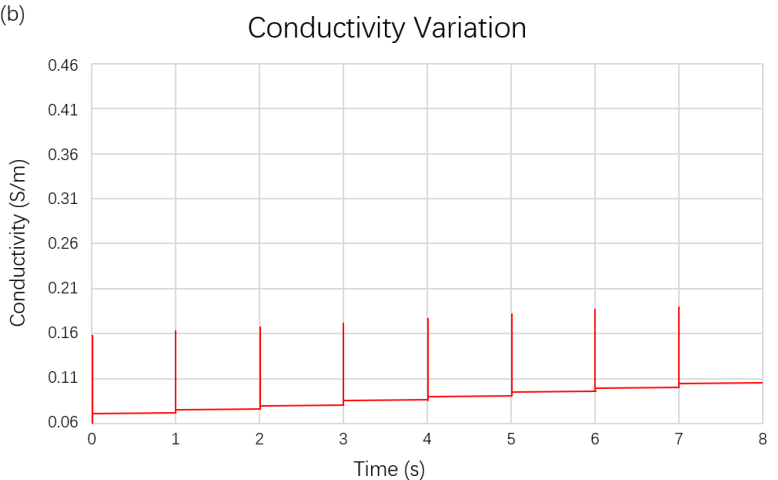

Fig. 3. Conductivity variation between two needle electrodes row under the action of 8 unipolar pulses whose frequency, pulse width, and E-field strength is $1 \mathrm{~Hz}, 2 \mathrm{~ms}$, and $3000 \mathrm{~V} / \mathrm{cm}$, respectively.

The results show that during the pulse transit time, the conductivity of the tissue gradually increased with time(Figures 2, 3, 4, 5). When the E-field intensity at the interest point is higher than the threshold, a change in conductivity occurs. The maximum conductivity variation at the electrode tip and between the electrode in the unipolar HVLF pulse is 0.44 and $0.18 \mathrm{~S} / \mathrm{m}$, respectively. These variations of the bipolar HVLF pulse are 0.205 and $0.16 \mathrm{~S} / \mathrm{m}$, respectively. It can be seen from the above results the conductivity variations of the unipolar HVLF pulse are greater than that of the bipolar HVLF pulse. The possible reason is that the maximum applied voltage of the unipolar pulse is larger than that of the bipolar pulse; i.e., $-V_{\max } / 2$ to $V_{\max } / 2$ vs. 0 to $V_{\max }$ [26]. And the variations in conductivity of the electrode tip is higher than the variations in conductivity between electrode rows. Because the E-field intensity at the electrode tip is higher.

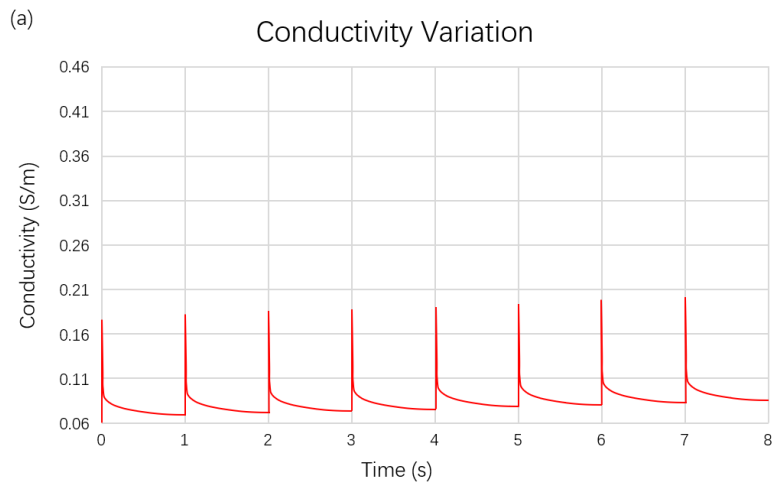

Fig. 4. Conductivity variation at the tip of needle electrodes under the action of 8 bipolar pulses whose frequency, pulse width, and E-field strength is $1 \mathrm{~Hz}, 2 \mathrm{~ms}$ and $3000 \mathrm{~V} / \mathrm{cm}$, respectively.

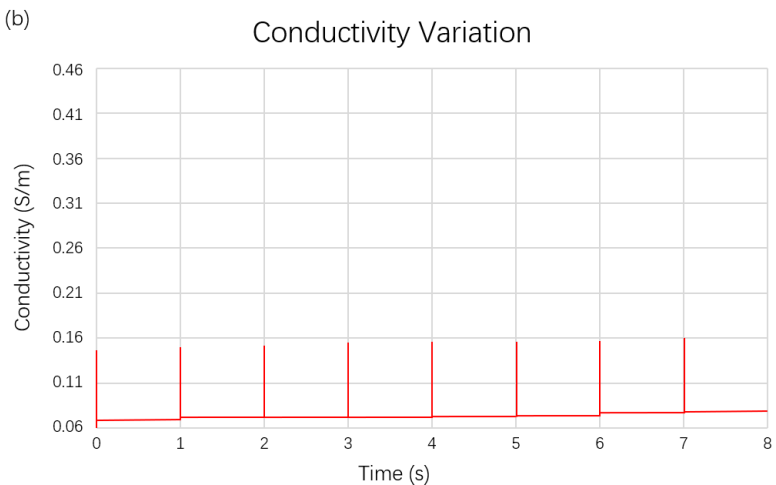

Fig. 5. Conductivity variation between two needle electrodes row under the action of 8 bipolar pulses whose the frequency, pulse width, and E-field strength is $1 \mathrm{~Hz}, 2 \mathrm{~ms}$ and $3000 \mathrm{~V} / \mathrm{cm}$, respectively.

\subsection{Simulation results for conductivity variation in LVHF}

During the pulse transmission time, the conductivity variation of the unipolar LVHF pulse at an E-field strength of $300 \mathrm{~V} / \mathrm{cm}$ is shown in Figure 6 and Figure 7. According to our data, the conductivity of the electrode tip has changed significantly. However, the conductivity between the electrodes hardly changed with the LVHF pulse.

\section{Conclusion}

Conductivity variation may have an important impact on temperature and E-field distribution and the result of treatment during IRE $[16,26]$. The LVHF pulse can only change the conductivity of the tissue at the tip of the 
electrode. The HVLF pulse will significantly change the conductivity in the tissue between the electrodes. Therefore, using LVHF pulses will reduce the error rate because the heat generated is low and the effect of muscle contraction is eliminated, and the effect of electrical conductivity variation on the E-field and temperature distribution can be ignored. In the clinic, LVHF pulse can be utilized as an electrical pulse for IRE which can be utilized as a treatment for cancer.

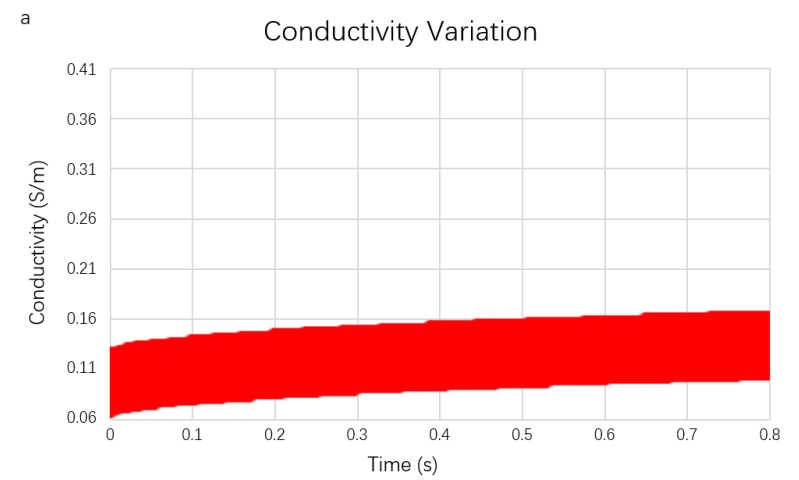

Fig. 6. Conductivity variation at the tip of the needle electrodes under the action of 3500 unipolar pulses whose frequency, pulse width, and E-field strength is $5.2 \mathrm{kHz}, 0.1 \mathrm{~ms}, 300 \mathrm{~V} / \mathrm{cm}$, respectively.

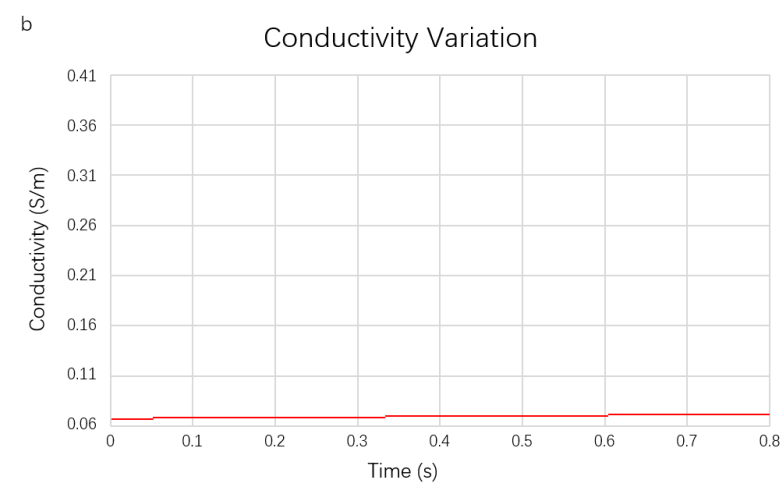

Fig. 7. Conductivity variation between the needle electrodes row under the action of 3500 unipolar pulses whose frequency, pulse width, and E-field strength is $5.2 \mathrm{kHz}, 0.1 \mathrm{~ms}, 300 \mathrm{~V} / \mathrm{cm}$, respectively.

\section{Acknowledgments}

This work was supported by grants from National Science and Technology Major Project (No. 2018ZX10301201), National Key Research and Development Program of China (No. 2019YFF0216302).

\section{References}

1. C. Jiang, R. V. Davalos, J. C. Bischof, IEEE T BIOMED ENG, 62, 4 (2015)

2. J. Lavee, G. Onik, P. Mikus, B. Rubinsky, HEART SURG FORUM, 10(2), 162 (2007)

3. B. Rubinsky, G, Onik, P. Mikus. CANCER RES TREAT, 6(1), 37 (2007)

4. E. Maor, A. Ivorra, J. J. Mitchell, B. Rubinsky, J VASC INTERV RADIOL, 21(11), 1708 (2010)
5. A. I. V. Ross, M. W. Griffiths, G. S. Mittal, H. C. Deeth, INT J FOOD MICROBIOL, 89(2-3), 125 (2003)

6. R. E. Neal, J. H. Rossmeisl, P. A. Garcia, O. I. Lanz, N. Henao-Guerrero, R. V. Davalos, J CLIN ONCOL, 29(13), 372 (2011)

7. T. P. Kingham, A. M. Karkar, M. I. D"Angelica, P. J. Allen, R. P. Dematteo, G. I. Getrajdman, J Am Coll Surg, 215(3), 379 (2012)

8. T. Froud, S. R. Venkat, K. J. Barbery, A. Gunjan, G. Narayanan, Tech Vasc Interv Radiol, 18(3), 140 (2015).

9. G. Narayanan, M. H. Doshi, CURR UROL REP, 17(2), 15 (2016)

10. P. Prejesh, H. David, R. C. G. Martin, Z. Zhuoli, PLOS ONE, 8(11), e76260 (2013).

11. C. B. Arena, M. B. Sano, J. H. Rossmeisl, J. L. Caldwell, R. V. Davalos, BIOMED ENG ONLINE, 10(1), 102 (2011)

12. J. P. Reilly, V. T. Freeman, W. D. Larkin, IEEE T BIO-MED ENG, 32(12), 1001 (1985)

13. C. B. Arena, M. B. Sano, J. H. Rossmeisl, J. L. Caldwell, R. V. Davalos, BIOMED ENG ONLINE, 10(1), 102 (2011)

14. D. Miklavcic, G. Pucihar, M. Pavlovec, S. Ribaric, M. Mali, A. Macek-Lebar, M. Petkovsek, J. Nastran, S. Kranjc, M. Cemazar, G. Sersa, BIOELECTROCHEMISTRY, 65(2), 121 (2005)

15. L. M. Mir, BIOELECTROCHEMISTRY, 53(1), 1 (2001)

16. S. Corovic, I. Lackovic, P. Sustaric, BIOMED ENG ONLINE, 12, 16 (2013)

17. E. M. Dunki-Jacobs, P. Philips, R. C. Martin, J Am Coll Surg, 218(2), 179 (2014).

18. M. G. Moisescu, M. Radu, E. Kovacs, L. M. Mir, T. Savopol, BBA-BIOMEMBRANES, 1828(2), 365 (2013)

19. M. Pavlin, M. Kanduser, M. Rebersek, G. Pucihar, D. Miklavcic, BIOPHYS J, 88(6), 4378 (2005)

20. D. Cukjati, D. Batiuskaite, D. Miklavcic, L. Mir, BIOELECTROCHEMISTRY, 70(2), 501 (2007).

21. S. Čorović, M. Pavlin, D. Miklavčič, BIOMED ENG ONLINE, 6, 37 (2007)

22. Z. Shankayi, S. M. Firoozabadi, Z. M. Hassan, Koomesh, 13(4), 486 (2012)

23. Z. Shankayi, S. M. P. Firoozabadi, Z. S. Hassan, J. Biomed. Phys. Eng. 3(3), 87 (2013)

24. M. B. Sano, R. E. Neal, P. A. Garcia, BIOMED ENG ONLINE, 9, 83 (2010)

25. P. A. Garcia, J. H. Rossmeisl Jr, R. E. Neal 2nd, T. L. Ellis, J. D. Olson, N. Henao-Guerrero, J. Robertson, R. V. Davalos, J MEMBRANE BIOL. 236(1), 127 (2010)

26. P. A. Garcia, R. V. Davalos, M. Damijan, R. Boris, PLOS ONE, 9(8), 1(2014) 